\title{
The Response of Nonlinear Single-Degree-of-Freedom Systems to Modulated High-Frequency Input
}

\author{
Atef F. El-Bassiouny \\ Faculty of Science, Mathematics Department, Benha University, Benha 13518, Egypt \\ Present address: P. O. Box 3137, Quassim, Onaizah, Kingdom of Saudi Arabia
}

Reprint requests to A.F. E.-B.; E-mail: atef_elbassiouny@yahoo.com

Z. Naturforsch. 61a, 541 - 555 (2006); received September 22, 2005

\begin{abstract}
In this paper we study the response of single-degree-of-freedom with cubic, quartic and quintic nonlinearities to an amplitude-modulated excitation whose carrier frequency is much higher than the natural frequency of the system. The only restriction on the amplitude modulation is that it contains frequencies much lower than the carrier frequency of the excitation. The method of multiple scales is used to derive two coupled first-order ordinary differential equations that describe the evolution of the amplitude and phase with damping, nonlinearities and resonances. The evolution equations are used to determine the steady-state motions, while representative frequency-response curves are presented for each resonance. Stability analysis of the amplitude and phase modulation equations for both cases are performed. The bending of the response curves leads to multi-valued solutions and hence to jump phenomena.
\end{abstract}

Key words: Nonlinear Oscillations; Modulation Equations; Resonances; Carrier Frequency; Fixed Points; Stability.

\section{Introduction}

In many applications, vibrations caused by rotating machinery are unavoidable. Often, supporting structures are isolated from this vibration by mounting the machinery on soft springs in order to form a supporting system with a natural frequency much lower than the frequency of operation of the machinery. The basic model of a vibration isolator as described above is a one-degree-of-freedom oscillator with a rotating unbalanced mass. If the system is linear, it is known to operator far from resonance and the force transmitted to the structure is small. If, however, nonlinearities are present in the support and the machine operates in a nonstationary manner, dangerous resonances would occur.

In most studies of the vibrations of weakly nonlinear systems, the system response as well as the excitation are at frequencies of comparable magnitude. In such systems, the nonlinearities usually act over several cycles of motion to generate changes in the amplitude and phase of the response. These changes are generally at a frequency much lower than the basic frequencies of the system. Perturbation methods, such as the method of averaging and multiple scales, explicitly take advantage of this fact in their formulation. Changes at high frequencies are said to be fast variations or to occur on a fast time scale, and changes at low frequencies are said to be slow variations or to occur on slow time scales.

Nayfeh et al. [1] showed that the single-mode discretization can lead to erroneous results for the response of a relief valve characterized by quadratic and cubic nonlinearities to primary resonance. They contrasted the results obtained by using a singlemode discretization with those obtained with the direct approach. Lu et al. [2] used the method of multiple scales to investigate subharmonic, superharmonic and quasiperiodic oscillations induced by vortices. Nayfeh et al. [3] compared the results obtained by using a single-mode discretization and direct treatment of several one-dimensional distributed-parameter systems with quadratic and cubic nonlinearities, including an Euler-Bernoulli beam resting on a nonlinear elastic foundation, surface waves in a rectangular container, and a relief valve. They found that the single-mode discretization may lead to erroneous results. Nayfeh and Nayfeh [4] investigated a singledegree-of-freedom system with cubic nonlinearities to an amplitude-modulated excitation whose carrier frequency is much higher than the natural frequency of the system. Thomsen [5] considered a string under 
similar conditions and used a sliding-mass nonlinear absorber. His studies revealed that, for moderate forcing amplitudes, successful suppression is possible. Anderson et al. [6] considered the effect of quadratic damping on the response of a cantilever beam. Deryugin et al. [7] developed an analytic approach to the problem of the stabilization behavior of dynamical systems by parametric perturbations. Yabuno [8] proposed a control law based on linear velocity feedback and linear and cubic velocity feedback. His studies demonstrated that nonlinear position feedback reduces the response amplitude in the parametric excitationresponse curve, while velocity feedback stabilizes the trivial solution in the frequency-response curve. Wilson [9] studied parametric spin resonance for a spinner with orbiting pivot. Zadeh and Galili [10] investigated a beam with tip mass under harmonic support motion. Nayfeh [11] proposed a scheme for constructing reduced-order models of distributed-parameter systems with quadratic and cubic nonlinearities. Approximate solution procedures based on reduced-order models via the Galerkin method are contrasted with direct application of the method of multiple scales to the governing partial differential equations and boundary conditions. Ge et al. [12] analyzed the nonlinear dynamic and chaos control of a damped satellite with a partially filled liquid. Raghothama and Narayanan [13] studied bifurcation and chaos in a geared rotor bearing system by an incremental harmonic balance method. Kreider and Nayfeh [14] analyzed the nonlinear single-mode responses in a fixed buckled beam. Lacarbonara and Nayfeh [15] investigated the experimental validation of reduction methods for nonlinear vibrations of distributed-parameter systems: analysis of a buckled beam. Oueini and Nayfeh [16] investigated the problem of suppressing the vibrations of a cantilever beam when subjected to primary and principal parametric resonances. The dynamics of the first mode are modeled with a second-order nonlinear ordinary differential equation, and a control law based on cubic velocity feedback is introduced. El-Bassiouny and Abdelhafez [17] investigated the predication of bifurcations for an external and parametric excited onedegree-of-freedom system with quadratic, cubic, and quartic nonlinearities. Maccari [18] studied the parametric resonance of a van der Pol oscillator under state feedback control with time delay. El-Dib [19] used the method of multiple scales to determne a third-order solution for a cubic nonlinear Mathieu equation. Eissa and El-Bassiouny [20] investigated analytical and nu- merical solutions of a nonlinear ship motion. Alsaif [21] investigated the dynamic response of a nonlinear semi-definite mechanical structure. It consists of a cantilever beam subjected to a harmonic excitation at the base; two hinged rigid plates are attached at the other end. El-Bassiouny and Eissa [22] studied the dynamics of a single-degree-of-freedom structure with quadratic, cubic and quartic nonlinearities to a harmonic resonance. $\mathrm{Wu}$ and $\mathrm{Li}$ [23] used two versions of multiple scales and Kryov-Bogoliubov-Mitropolsky (KBM) methods to obtain the asymptotic solutions of a class of strongly nonlinear oscillators with slowly varying parameters. A comparison of the two methods is made to show that the multiple scales method is equivalent to the KBM method for the first-order approximation. Elhefnawy and El-Bassiouny [24] analyzed the nonlinear stability and chaos in electrohydrodynamics. Maccari [25] used the asymptotic perturbation method to study the parametrically excited and weakly damped Boussinesq equation in an infinite wall. El-Bassiouny [26] used the method of multiple scales to investigate principal parametric resonances of nonlinear mechanical system with two-frequency and self-excitations. Cao [27] studied the primary resonant optimal control for homoclinic bifurcations in single-degree-offreedom nonlinear oscillators. Jing and Wang [28] analyzed complex dynamics in a Duffing system with two external forcings. Pakemirli et al. [29] analyzed the response of a transversely excited suspended cable with a one-to-one internal resonance between the third inplane and out-plane modes. In this case, two modes are retained in the discretization, and yet the discretization results are erroneous for some excitation amplitudes and frequencies. Maccari [30] analyzed a modulated motion and infinite-period bifurcation for two nonlinearly coupled and parametrically excited van der Pol oscillators. El-Bassiouny [31] analyzed simultaneous primary resonances of a two-degree-of-freedom system with quadratic, cubic and quartic nonlinearities to external excitations having the frequencies $\Omega_{1}$ and $\Omega_{2}$. Elnaggar and El-Bassiouny [32] used the Tondl technique to analyze theoretical investigations of periodic and nonperiodic combination resonances of a system of rods under vertical and horizontal kinematic excitations. Bi [33] studied the dynamical analysis of two coupled parametrically excited van der Pol oscillators. El-Bassiouny and Eissa [34] used the method of multiple scales and the generalized synchronization method to investigate the sub-harmonic resonance of two-degree-of-freedom systems with quadratic and 
cubic nonlinearities to multi-frequency parametric excitations in the presence of two-to-one internal resonance. El-Bassiouny [35] investigated the dynamic stability of complicated motions of a vessel; in the following regular waves are investigated when the frequency in the pitch is nearly twice the frequency in the roll. We used the method of multiple scales to determine four first-order nonlinear ordinary differential equations that govern the modulation of the amplitudes and phases when the encounter frequency is near either the pitch or the roll natural frequency. El-Bassiouny [36] studied an approach for implementing an active nonlinear vibration absorber. The strategy exploits the saturation phenomenon that is exhibited by multi-degreeof-freedom systems with cubic nonlinearities possessing one-to-one internal resonance. The proposed technique consists of introducing a second-order controller and coupling it to the plant through a sensor and an actuator where both the feedback and control signals are cubic. El-Bassiouny [37] investigated the nonlinear interaction of a three-degree-of-freedom structural model subjected to external excitation. The nonlinearity of the system results in different critical regions of internal resonance, and this has a significant effect on the response.

In this paper we study the response of a singledegree-of-freedom system with cubic, quartic and quintic nonlinearities to an amplitude-modulated highfrequency input. It was shown that, when a nonlinear system is subjected to an external excitation centered at a frequency much higher than its own natural frequency, the system averages the excitation, smoothing away the high-frequency carrier but leaving behind any low-frequency variations. Under some conditions, these low-frequency components may excite low-frequency motions.

\section{Formulation of the Problem}

We consider a single-degree-of-freedom oscillator with cubic, quartic and quintic nonlinearities given by

$$
\begin{aligned}
& \frac{\mathrm{d}^{2} X}{\mathrm{~d} t^{2}}+2 \mu \omega \frac{\mathrm{d} X}{\mathrm{~d} t}+\omega^{2} X \\
& +\omega^{2}\left(\alpha X^{3}+\beta X^{4}+\gamma X^{5}\right)=F(\omega t) \cos t,
\end{aligned}
$$

where $t$ is nondimensionalized so that the carrier frequency of the excitation is unity, the natural frequency $\omega$ of the system is small, $\mu$ is the damping factor, $\alpha, \beta$ and $\gamma$ are the nonlinear coefficients. To show explicitly that the amplitude of the excitation $F$ is slowly varying, we write it as a function of $\omega t$.

\section{Method of Solution}

We use the method of multiple scales $[38,39]$ to determine a second-order expansion of the solutions of (1). We begin by introducing the new independent variables $T_{0}=t, T_{1}=\omega t$, and $T_{2}=\omega^{2} t$ which characterize, respectively, the motion near the excitation frequency, near the natural frequency of the system and at lower frequencies. Using the chain rule, we write the time derivative in terms of $T_{i}$ as

$$
\frac{\mathrm{d}}{\mathrm{d} t}=\frac{\partial}{\partial T_{0}}+\omega \frac{\partial}{\partial T_{1}}+\omega^{2} \frac{\partial}{\partial T_{2}}
$$

The response of the system, described by (1), can be expressed in the form

$$
\begin{aligned}
X(t ; \varepsilon)= & X_{0}\left(T_{0}, T_{1}, T_{2}, \ldots\right)+\omega X_{1}\left(T_{0}, T_{1}, T_{2}, \ldots\right) \\
& +\omega^{2} X_{2}\left(T_{0}, T_{1}, T_{2}, \ldots\right)+\ldots
\end{aligned}
$$

Inserting (2) and (3) into (1), and equating coefficients of like power of $\omega$, we get

$$
\begin{aligned}
\frac{\partial^{2} X_{0}}{\partial T_{0}^{2}}= & F\left(T_{1}\right) \cos T_{0}, \\
\frac{\partial^{2} X_{1}}{\partial T_{0}^{2}}= & -2\left(\frac{\partial^{2} X_{0}}{\partial T_{0} \partial T_{1}}+\mu \frac{\partial X_{0}}{\partial T_{0}}\right), \\
\frac{\partial^{2} X_{2}}{\partial T_{0}^{2}}=- & {\left[\left(\frac{\partial^{2} X_{0}}{\partial T_{1}^{2}}+2 \frac{\partial^{2} X_{0}}{\partial T_{0} \partial T_{2}}\right)\right.} \\
& -2 \frac{\partial^{2} X_{1}}{\partial T_{0} \partial T_{1}}+2 \mu\left(\frac{\partial X_{0}}{\partial T_{1}}+2 \frac{\partial X_{1}}{\partial T_{0}}\right) \\
& \left.+X_{0}+\alpha X_{0}^{3}+\beta X_{0}^{4}+\gamma X_{0}^{5}\right] .
\end{aligned}
$$

The general solution of (4) can be given in the form

$$
X_{0}=A\left(T_{1}\right)+B\left(T_{1}\right) T_{0}+F\left(T_{1}\right) \cos T_{0} .
$$

We require that the response be free of secular terms and therefore set $B=0$. We note that, in (7), the term $F\left(T_{1}\right) \cos T_{0}$ represents the direct response to the high-frequency external forcing function and the term $A\left(T_{1}\right)$, which will be determined at higher-order, captures low-frequency motion near the natural frequency 
of the system. We will find that, through the nonlinearity, the high-frequency motion can influence the lowfrequency motion, causing resonant responses to occur under certain conditions.

Next, we substitute the expression for $X_{0}$ given in (7) into (5), and solving for $X_{1}$ yields

$$
X_{1}=2\left(F^{\prime}+\mu F\right) \sin T_{0},
$$

where the prime indicates the derivative to $T_{1}$. Substituting (7) and (8) into (6), we obtain

$$
\begin{aligned}
\frac{\partial^{2} X_{2}}{\partial T_{0}^{2}}= & -A^{\prime \prime}-2 F^{\prime \prime} \cos T_{0}-\left(A-F \cos T_{0}\right) \\
& -2 \mu\left[A^{\prime}+F^{\prime} \cos T_{0}+2\left(\mu F+F^{\prime}\right) \cos T_{0}\right] \\
& -\alpha\left(A-F \cos T_{0}\right)^{3}-\beta\left(A-F \cos T_{0}\right)^{4} \\
& -\gamma\left(A-F \cos T_{0}\right)^{5}
\end{aligned}
$$

Secular terms are those which cause $X_{2}$ to grow without bound as $T_{0} \rightarrow \infty$. In this case, the secular terms are the slowly varying terms. Eliminating them, we obtain

$$
\begin{aligned}
& A^{\prime \prime}+2 \mu A^{\prime}+\left(1+\frac{3}{2} \alpha F^{2}+\frac{15}{8} \gamma F^{4}\right)+\alpha A^{3} A \\
& +\beta A^{4} \gamma A^{5}+\beta\left(3 A^{2} F^{2}+\frac{3}{8} F^{4}\right) A+5 \gamma A^{3} F^{2}=0 .
\end{aligned}
$$

Hence, to the second approximation, $X$ is given by

$$
X=A-F \cos t+2 \omega\left(F^{\prime}+\mu F\right) \sin t+\ldots .
$$

Thus, the response of the system consists of components at the frequency of the excitation and a lowfrequency component governed by (11). We note that the amplitude of the high-frequency excitation appears as a parametric excitation on the low-frequency component of the response, and therefore a modulated high-frequency excitation can cause resonant responses.

\section{Response to Harmonically Modulated Excitation}

In this section we study the case where the excitation amplitude undergoes a simple-harmonic modulation. We therefore let

$$
F=f+g \cos \Omega T_{1}
$$

We note that the excitation $F \cos t$ can in this case be written as a sum of harmonics as

$$
F \cos t=f \cos t+\frac{1}{2} g[\cos (1+\Omega \omega) t+\cos (1-\Omega \omega) t] .
$$

Thus, the modulated excitation studied in this case can be viewed as a three-frequency excitation and the results can be interpreted as such.

\subsection{Weak Nonlinearity}

In this section we use (13) to study the case of smaller values of $\alpha, \beta, \gamma, \mu$, and $F$. Thus the same results can be obtained by performing a two-parameter multiple scales expansion of (1). To this end we introduce a small dimensionless parameter $\varepsilon$ to characterize the smallness of $\mu, \alpha, \beta, \gamma$, and rewrite (1) as

$$
\begin{aligned}
& \frac{\mathrm{d}^{2} X}{\mathrm{~d} t^{2}}+2 \varepsilon \mu \omega \frac{\mathrm{d} X}{\mathrm{~d} t}+\omega^{2} X \\
& +: \varepsilon \omega^{2}\left(\alpha X^{3}+\beta X^{4}+\gamma X^{5}\right)=F(\omega t) \cos t .
\end{aligned}
$$

We assume that $\varepsilon \ll \omega$ but $\omega^{2} \ll \varepsilon$ and seek a twoparameter multiple scales expansion in the form

$$
\begin{aligned}
X(t ; \varepsilon, \omega)= & X_{0}\left(T_{0}, T_{1}, \tau_{1}, T_{2}\right)+\omega X_{1}\left(T_{0}, T_{1}, \tau_{1}, T_{2}\right) \\
& +\varepsilon V_{1}\left(T_{0}, T_{1}, \tau_{1}, T_{2}\right)+\varepsilon \omega V_{2}\left(T_{0}, T_{1}, \tau_{1}, T_{2}\right) \\
& +\omega^{2} X_{2}\left(T_{0}, T_{1}, \tau_{1}, T_{2}\right) \\
& +\varepsilon \omega^{2} V_{3}\left(T_{0}, T_{1}, \tau_{1}, T_{2}\right),
\end{aligned}
$$

where

$$
T_{0}=t, \quad T_{1}=\omega t, \quad T_{2}=\omega^{2} t, \text { and } \tau_{1}=\varepsilon \omega t=\varepsilon T_{1} .
$$

In terms of the new time scales, the time derivative becomes

$$
\begin{aligned}
\frac{\mathrm{d}}{\mathrm{d} t}= & \frac{\partial}{\partial T_{0}}+\omega \frac{\partial}{\partial T_{1}}+\varepsilon \omega \frac{\partial}{\partial \tau_{1}}+\omega^{2} \frac{\partial}{\partial T_{2}}+\ldots \\
\frac{\mathrm{d}^{2}}{\mathrm{~d} t^{2}}= & \frac{\partial^{2}}{\partial T_{0}^{2}}+2 \omega \frac{\partial^{2}}{\partial T_{0} \partial T_{1}}+2 \varepsilon \omega \frac{\partial^{2}}{\partial T_{0} \partial \tau_{1}} \\
& +2 \omega^{2} \frac{\partial^{2}}{\partial T_{0} \partial T_{2}}+\omega^{2} \frac{\partial^{2}}{\partial T_{0}^{2}}+2 \varepsilon \omega^{2} \frac{\partial^{2}}{\partial T_{1} \partial \tau_{1}} \\
& +\ldots
\end{aligned}
$$

Inserting (15) and (16) into (14) and equating the coefficients of like power of $\varepsilon^{0}, \omega, \varepsilon \omega, \omega^{2}$, and $\varepsilon \omega^{2}$ on both sides, we obtain: 
order of $\varepsilon^{0}$ :

$$
\frac{\partial^{2} X_{0}}{\partial T_{0}^{2}}=F\left(T_{1}\right) \cos T_{0}
$$

order of $\omega$ :

$$
\frac{\partial^{2} X_{1}}{\partial T_{0}^{2}}=-2 \frac{\partial^{2} X_{0}}{\partial T_{0} \partial T_{1}}
$$

order of $\varepsilon$ :

$$
\frac{\partial^{2} V_{1}}{\partial T_{0}^{2}}=0
$$

order of $\varepsilon \omega$ :

$$
\frac{\partial^{2} V_{2}}{\partial T_{0}^{2}}=\frac{\partial^{2} X_{0}}{\partial T_{0} \partial \tau_{1}}-2 \frac{\partial^{2} V_{1}}{\partial T_{0} \partial T_{1}}-2 \mu \frac{\partial X_{0}}{\partial T_{0}}
$$

order of $\omega^{2}$ :

$$
\frac{\partial^{2} X_{2}}{\partial T_{0}^{2}}=-X_{0}-\frac{\partial^{2} X_{0}}{\partial T_{1}^{2}}-2 \frac{\partial^{2} X_{1}}{\partial T_{0} \partial T_{1}}-\frac{\partial^{2} X_{0}}{\partial T_{0} \partial T_{2}}
$$

order of $\varepsilon \omega^{2}$ :

$$
\begin{aligned}
\frac{\partial^{2} V_{3}}{\partial T_{0}^{2}}= & -V_{1}-\frac{\partial^{2} V_{1}}{\partial T_{1}^{2}}-2 \frac{\partial^{2} V_{2}}{\partial T_{0} \partial T_{1}}-\frac{\partial^{2} X_{1}}{\partial T_{0} \partial \tau_{1}} \\
& -2\left(\frac{\partial^{2} V_{1}}{\partial T_{0} \partial T_{2}}+\frac{\partial^{2} X_{0}}{\partial T_{1} \partial \tau_{1}}+\mu \frac{\partial X_{0}}{\partial T_{1}}+\mu \frac{\partial X_{1}}{\partial T_{0}}\right) \\
& -\alpha X_{0}^{3}-\beta X_{0}^{4}-\gamma X_{0}^{5}
\end{aligned}
$$

The solution of (17) is given by

$$
X_{0}=A_{0}\left(T_{1}, \tau_{1}, T_{2}\right)-F\left(T_{1}\right) \cos T_{0}
$$

Using (23) in (18) and solving the resulting equation, we get

$$
X_{1}=2 F^{\prime} \sin T_{0},
$$

where the prime denotes the derivative with respect to $T_{1}$. The solution of (19) can be expressed as

$$
V_{1}=A_{1}\left(T_{1}, \tau_{1}, T_{2}\right)
$$

Substituting (23) and (25) into (20) and solving the resulting equation, we get

$$
V_{2}=2 \mu F \sin T_{0}
$$

Inserting (23) and (25) into (21) yields

$$
\frac{\partial^{2} X_{2}}{\partial T_{0}^{2}}=-\frac{\partial^{2} A_{0}}{\partial T_{1}^{2}}-A_{0}+F \cos T_{0}+3 F^{\prime \prime} \cos T_{0}
$$

Eliminating the terms in (27) that produce secular terms yields

$$
\frac{\partial^{2} A_{0}}{\partial T_{1}^{2}}+A_{0}=0
$$

Then, the solution of (27) can be expressed as

$$
X_{2}=-\left(F+3 F^{\prime \prime}\right) \cos T_{0} .
$$

Also, the solution of (28) becomes

$$
A_{0}=G\left(\tau_{1}\right) \exp \left(\mathrm{i} T_{1}\right)+\bar{G}\left(\tau_{1}\right) \exp \left(-\mathrm{i} T_{1}\right),
$$

where $\bar{G}$ is the complex conjugated of $G$. To determine the dependence of $A_{0}$ on $\tau$, we must carry the expansion to higher-order than in (15). Using (22) - (26) in (22) and eliminating the terms that produce secular terms, we get

$$
\frac{\partial^{2} A_{1}}{\partial T_{1}^{2}}+A_{1}=-2 \frac{\partial^{2} A_{0}}{\partial T_{1} \partial \tau_{1}}-2 \mu \frac{\partial A_{0}}{\partial T_{1}}-\alpha\left(A_{0}^{2}+\frac{3}{2} \alpha F^{2} A_{0}\right)-\beta\left(A_{0}^{4}+3 A_{0}^{2} F^{2}+\frac{3}{8} F^{4}\right)-\gamma\left(A_{0}^{5}+5 A_{0}^{3} F^{2}+\frac{15}{8} F^{4} A_{0}\right) .
$$

Substituting (30) into (31) and using the specific form for $F$ given in (13), we obtain

$$
\begin{gathered}
\frac{\partial^{2} A_{1}}{\partial T_{0}^{2}}+A_{1}=-\left[2 \mathrm{i}\left(G^{\prime}+\mu G\right)+\frac{3}{2} \alpha G\left(f^{2}+\frac{1}{2} g^{2}\right)+\frac{15}{8} \alpha G\left(f^{4}+3 f^{2} g^{2}+\frac{3}{16} g^{4}\right)\right. \\
\left.+3 \alpha G^{2} \bar{G}+10 \gamma G^{3} \bar{G}^{2}+15 \gamma G f^{4}+15 \gamma f^{2} G^{3} \bar{G}^{2}\right] \exp \left(\mathrm{i} \tau_{0}\right) \\
-\frac{15}{8} \alpha \bar{G}^{2}\left[\left(f^{3} g+\frac{3}{4} f g^{3}\right) \exp g\left\{\mathrm{i}\left(\Omega T_{1}-\tau_{0}\right)\right\}-\left(\frac{3}{2} f^{2} g^{2}+\frac{5}{32} g^{4}\right) \exp \left\{\mathrm{i}\left(2 \Omega T_{1}-\tau_{0}\right)\right\}\right.
\end{gathered}
$$




$$
\begin{gathered}
\left.+\frac{1}{2} f g^{3} \exp \left\{\mathrm{i}\left(3 \Omega T_{1}-1\right) \tau_{0}\right\}+\frac{1}{16} g^{4} \exp \left\{\mathrm{i}\left(4 \Omega T_{1}-2\right) \tau_{0}\right\}\right] \\
-\frac{3}{8} \beta\left[\left(2 f^{3} g+\frac{3}{4} f g^{3}\right) \exp \left(\mathrm{i} \Omega T_{1}\right)+\left(\frac{3}{2} f^{2} g^{2}+\frac{5}{32} g^{4}\right) \exp \left(2 \mathrm{i} \Omega T_{1}\right)+\frac{1}{2} f g^{3} \exp \left(3 \mathrm{i} \Omega T_{1}\right)+\frac{1}{16} g^{4} \exp \left(4 \mathrm{i} \Omega T_{1}\right)\right] \\
-5 \gamma\left[G \bar{G}^{2} \exp \left\{\mathrm{i}\left(\Omega T_{1}-\tau_{0}\right)\right\}+\bar{g}^{2} \exp \left\{\mathrm{i}\left(\Omega T_{1}-3 \tau_{0}\right)\right\}+\frac{3}{4} G \bar{G}^{2} g^{2} \exp \left\{\mathrm{i}\left(2 \Omega T_{1}-\tau_{0}\right)\right\}\right. \\
\left.+\frac{1}{4} \bar{G}^{2} g^{2} \exp \left\{\mathrm{i}\left(2 \Omega T_{1}-3 \tau_{0}\right)\right\}\right]+\mathrm{NST}+\mathrm{cc}
\end{gathered}
$$

where NST denotes that the terms do not produce secular terms and cc denotes the complex conjugate of the proceeding terms.

Then, the deduced resonance cases from (32) are as follows:

(i) primary resonance: $\Omega \cong 1$;

(ii) sub-harmonic resonance: $a-\Omega \cong 2, b-\Omega \cong$ $3, c-\Omega \cong 4$;

(iii) superharmonic resonance: $a-2 \Omega \cong 1, b-$ $3 \Omega \cong 1, c-4 \Omega \cong 1$;

(iv) sub-superharmonic resonance: $3 \Omega \cong 2$.

In the following sections, we consider two cases of resonances.

\subsection{First Case: Superharmonic Resonance}

In this section, we consider the case of superharmonic resonance $\left(4 \Omega \cong \tau_{0}\right)$ and therefore set $4 \Omega=$ $\tau_{0}+\varepsilon \sigma$. Eliminating secular terms from (32), we obtain the following modulation equation governing $G$ :

$$
\begin{aligned}
& 2 \mathrm{i}\left(G^{\prime}+\mu G\right)+\frac{3}{2} \alpha G\left(f^{2}+\frac{1}{2} g^{2}\right) \\
& +\frac{15}{16} \alpha G\left(f^{4}+3 f^{2} g^{2}+\frac{3}{16} g^{4}\right)+3 \alpha G^{2} \bar{G} \\
& +10 \gamma G^{3} \bar{G}^{2}+15 \gamma G f^{4}+15 \gamma f^{2} G^{3} \bar{G}^{2} \\
& -\frac{3}{128} \beta g^{4} \exp \left(4 \mathrm{i} \Omega T_{1}\right)=0 .
\end{aligned}
$$

Next, we separate the modulation equation into the real and imaginary part by writing $G$ in terms of an amplitude and phase as

$$
G=\frac{1}{2} r \exp (\mathrm{i} \beta) \text {. }
$$

Inserting (34) into (33), we obtain the autonomous system of equations

$$
r^{\prime}=-\mu r-\frac{3}{128} \beta g^{4} \sin \theta_{1}
$$

$$
\begin{aligned}
r \beta^{\prime}= & \frac{3}{4} \alpha r\left(f^{2}+\frac{1}{2} g^{2}\right) \\
& +\frac{15}{16} \alpha r\left(f^{4}+3 f^{2} g^{2}+\frac{3}{16} g^{4}\right) \\
& +\frac{3}{8} \alpha r^{3}+\frac{5}{16} \gamma r^{5}+\frac{15}{8} \gamma r^{3}+\frac{3}{128} \beta g^{4} \cos \theta_{1}
\end{aligned}
$$

where

$$
\theta_{1}=\sigma_{1}-\beta
$$

The steady-state response can be obtained by putting $r^{\prime}=0$ and $\beta^{\prime}=0$ in (35) and (36). Thus, the steady-state amplitude and phase are governed by the following equations:

$$
\begin{aligned}
& -\mu r-\frac{3}{128} \beta g^{4} \sin \theta_{1}=0, \\
& \sigma_{1} r-\frac{3}{4} \alpha r\left(f^{2}+\frac{1}{2} g^{2}\right) \\
& -\frac{15}{16} \alpha r\left(f^{4}+3 f^{2} g^{2}+\frac{3}{16} g^{4}\right)-\frac{3}{8} \alpha r^{3} \\
& -\frac{5}{16} \gamma r^{5}-\frac{15}{8} \gamma r^{3}-\frac{3}{128} \beta g^{4} \cos \theta_{1}=0,
\end{aligned}
$$

which lead to the following frequency-response equation:

$$
\begin{aligned}
\mu^{2}+[ & \frac{1}{3} \sigma_{2}-\frac{3}{4} \alpha\left(f^{2}+\frac{1}{2} g^{2}\right) \\
& -\frac{15}{16} \alpha\left(f^{4}+3 f^{2} g^{2}+\frac{3}{16} g^{4}\right)-\frac{3}{8} \alpha r^{2} \\
& \left.-\frac{5}{16} \gamma r^{4}-\frac{15}{8} \gamma r^{2}\right]^{2}+\left(\frac{3}{128}\right)^{2} \beta^{2} g^{8}=0 .
\end{aligned}
$$

Let nontrivial solutions of (40) have a small variation from the steady-state solutions $r_{0}$ and $\theta_{10}$, so that

$$
r=r_{0}+r_{1}, \quad \theta_{1}=\theta_{10}+\theta_{11}
$$


where $r_{1}$ and $\theta_{11}$ are assumed to be infinitesimal. Substituting (41) into (35) and (36) and keeping only linear terms in the perturbation quantities, using (38) and (39), we obtain

$$
\begin{aligned}
r_{1}^{\prime}= & -\mu r_{1}-\frac{3}{16} \beta f g^{3} \cos \theta_{10} \theta_{11}, \\
\theta_{11}^{\prime}= & {\left[\frac{\sigma_{1}}{r_{0}}-\frac{3 \alpha}{16 r_{0}}\left(4 f^{2}+2 g^{2}+5 f^{4}+15 f^{2} g^{2}\right.\right.} \\
& \left.\left.+\frac{3}{16} g^{4}\right)+\frac{9}{8}(\alpha+5 \gamma) r_{0}+\frac{15 \alpha}{8} r_{0}^{3}\right] r_{1} \\
& +\frac{3}{128 r_{0}} \beta g^{4} \sin \theta_{10} \theta_{11} .
\end{aligned}
$$

Since (42) and (43) represent a system of linear first-order equations with constant coefficients of $r_{1}$ and $\theta_{11}$, one can seek their solutions in the form

$$
r_{1}=r^{*} \exp \left(\lambda T_{1}\right), \quad \theta_{11}=\theta^{*} \exp \left(\lambda T_{1}\right),
$$

where $r^{*}$ and $\theta^{*}$ are real constants. Inserting (44) into (42) and (43), one gets for a nontrivial solution of $r^{*}$ and $\theta^{*}$ the equation

$$
\begin{aligned}
& \lambda^{2}+\left[\mu-\frac{3}{16 r_{0}} \beta f g^{3} \sin \theta_{10}\right] \lambda \\
& +\frac{3}{128} \beta g^{4} \cos \theta_{10}\left\{\frac{\sigma_{1}}{r_{0}}-\frac{3 \alpha}{16 r_{0}}\left(4 f^{2}+2 g^{2}\right.\right. \\
& \left.\left.+5 f^{4}+15 f^{2} g^{2}+\frac{3}{16} g^{4}\right)\right\}-\frac{3 \zeta}{128 r_{0}} \beta g^{4} \sin \theta_{10}=0,
\end{aligned}
$$

which is characteristic for the stability criterion. The necessary and sufficient condition for stability can be derived from the Hurwitz criteria for stability, which shows that the real parts of all the roots of the characteristic equation (45) are negative.

\subsection{Second Case: Sub-Superharmonic Resonance}

In this section we consider the case of superharmonic resonance of $3 \Omega \cong 2$ and therefore set $3 \Omega=$ $2 \tau_{0}+\varepsilon \sigma$. Eliminating secular terms from (32), we obtain the following modulation equation governing $G$ :

$$
\begin{aligned}
& 2 \mathrm{i}\left(G^{\prime}+\mu G\right)+\frac{3}{2} \alpha G\left(f^{2}+\frac{1}{2} g^{2}\right) \\
& +\frac{15}{8} \alpha G\left(f^{4}+3 f^{2} g^{2}+\frac{3}{16} g^{4}\right)+3 \alpha G^{2} \bar{G} \\
& +10 \gamma G^{3} \bar{G}^{2}+15 \gamma G f^{4}+15 \gamma f^{2} G^{3} \bar{G}^{2} \\
& -\frac{15}{16} \alpha \bar{G}^{2} f g^{3} \exp \left\{\mathrm{i}\left(3 \Omega T_{1}-1\right) \tau_{0}\right\}=0
\end{aligned}
$$

Inserting (34) into (46), we obtain the autonomous system of equations

$$
\begin{aligned}
r^{\prime} & =-\mu r-\frac{15}{16} \alpha r^{2} f g^{3} \sin \theta_{2}, \\
r \beta^{\prime}= & \frac{3}{4} \alpha r\left(f^{2}+\frac{1}{2} g^{2}\right)+\frac{15}{16} \alpha r\left(f^{4}+3 f^{2} g^{2}+\frac{3}{16} g^{4}\right) \\
& +\frac{3}{8} \alpha r^{3}+\frac{5}{16} \gamma r^{5}+\frac{15}{8} \gamma r^{3}+\frac{15}{64} \gamma r^{2} f g^{3} \cos \theta_{2},
\end{aligned}
$$

where

$$
\theta_{2}=\sigma_{2}-3 \beta
$$

For the stationary state there exist two possibilities, $r=$ 0 and $r \neq 0$. In the case of $r \neq 0$ the frequency-response equation becomes

$$
\begin{aligned}
\mu^{2}+[ & \frac{1}{3} \sigma_{2}-\frac{3}{4} \alpha\left(f^{2}+\frac{1}{2} g^{2}\right) \\
& -\frac{15}{16} \alpha\left(f^{4}+3 f^{2} g^{2}+\frac{3}{16} g^{4}\right)-\frac{3}{8} \alpha r^{2} \\
& \left.-\frac{5}{16} \gamma r^{4}-\frac{15}{8} \gamma r^{2}\right]^{2}-\frac{225}{3096} \alpha^{2} r^{2} f^{2} g^{6}=0 .
\end{aligned}
$$

To determine the stability of the nontrivial steady-state responses given by (47) and (48), let

$$
r=r_{0}+r_{1}, \quad \theta_{2}=\theta_{20}+\theta_{21},
$$

where $r_{0}$ and $\theta_{20}$ are the steady-state equations. Substituting (51) into (47) and (48), using steady-state equations and keeping only the linear terms in $r_{1}$ and $\theta_{21}$, we get

$$
\begin{aligned}
r_{1}^{\prime}= & -\left(\mu+\frac{15}{32} \alpha f g^{3} \sin \theta_{20}\right) r_{1} \\
& -\frac{15}{16} \alpha f g^{3} r_{0}^{2} \cos \theta_{20} \theta_{21},
\end{aligned}
$$

$$
\begin{aligned}
& \theta_{11}^{\prime}= \\
& \quad\left[\frac{\sigma_{2}}{r_{0}}-\frac{9 \alpha}{16 r_{0}}\left(4 f^{2}+2 g^{2}+5 f^{4}+15 f^{2} g^{2}+\frac{15}{16} g^{4}\right)\right. \\
& \left.+\frac{27}{8}(\alpha+3 \gamma) r_{0}+\frac{15 \alpha}{16} \gamma r_{0}^{3}+\frac{45}{32} \alpha f g^{3} \cos \theta_{20}\right] r_{1} \\
& \quad+\frac{45}{64} \alpha f g^{3} r_{0} \sin \theta_{20} \theta_{21} .
\end{aligned}
$$


Table 1. Ranges of the different parameters investigated.

\begin{tabular}{lll}
\hline Figure number & Investigated parameter & Range \\
\hline $1,2,3$ & Damping factor $\mu$ & 0.01 to 1.5 \\
4,5 & Coefficient of cubic nonlinearity $\alpha$ & 0.001 to 0.3 \\
6 & Coefficient of quartic nonlinearity $\beta$ & 0.2 to 10 \\
7 & Coefficient of quintic nonlinearity $\gamma$ & 0.5 to 15 \\
8,9 & Coefficient of external excitation $f$ & 0.01 to 5 \\
10 & Coefficient of external excitation $g$ & 2 to 8 \\
\hline
\end{tabular}

Stability of these fixed point solutions can be determined from the eigenvalues of the Jacobian matrix of (52) and (53).

\section{Numerical Results of the Frequency Response Curves}

In this section the numerical solutions of the frequency-response equations (40) and (50) are studied. These are nonlinear algebraic equations in the amplitude $r$. The results are plotted in Figs. 1-18, which present the variation of the amplitude $r$ whith the detuning parameters $\sigma_{1}$ and $\sigma_{2}$ for the first and second case, respectively.

Figures 1-10 represent frequency-response curves of the first case (superharmonic resonance). The effect of the different parameters on the system are investigated. In Table 1 the investigated ranges of the considered parameters are summarized.

In Fig. 1 we note that the response amplitude multivalued continuous curve and consists of two branches,

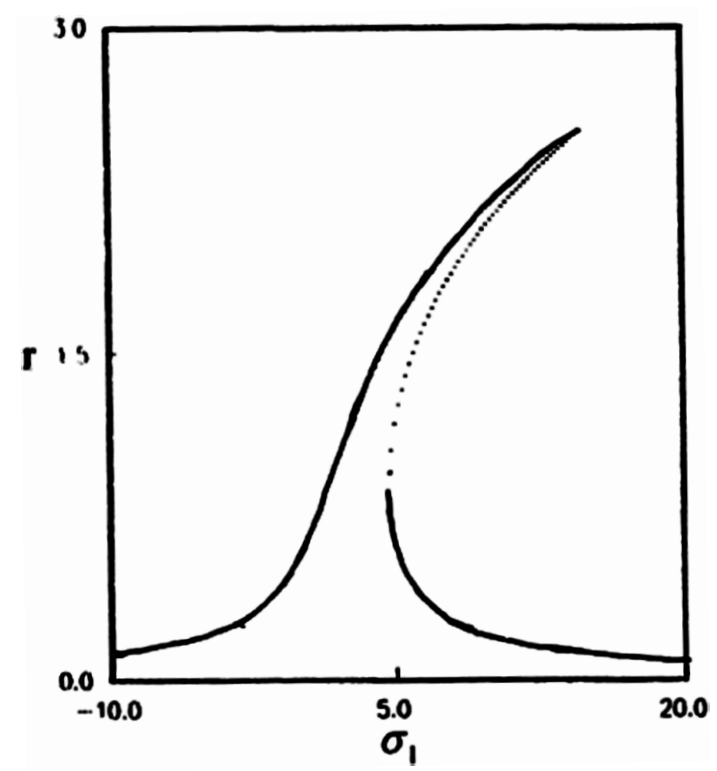

Fig. 1. Frequency-response curve for superharmonic resonance for the parameters $\mu=0.3, \alpha=0.01, \beta=0.2, \gamma=$ $0.5, f=1$ and $g=2$. (-) Stable and ( $\cdots)$ unstable.

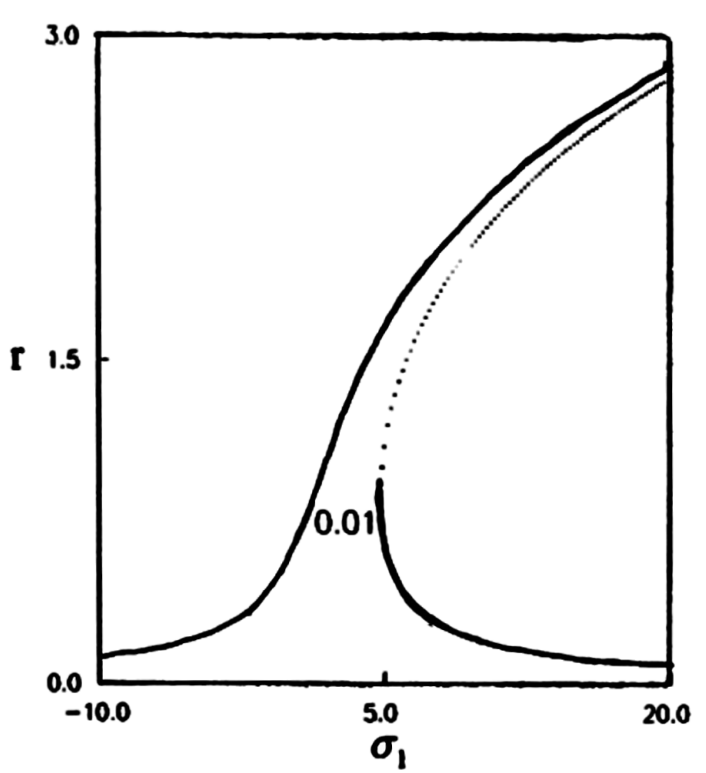

Fig. 2. Frequency-response curve for superharmonic resonance for decreasing the damping factor $\mu$ on the response when $\mu=0.01$. (-) Stable and $(\cdots)$ unstable.

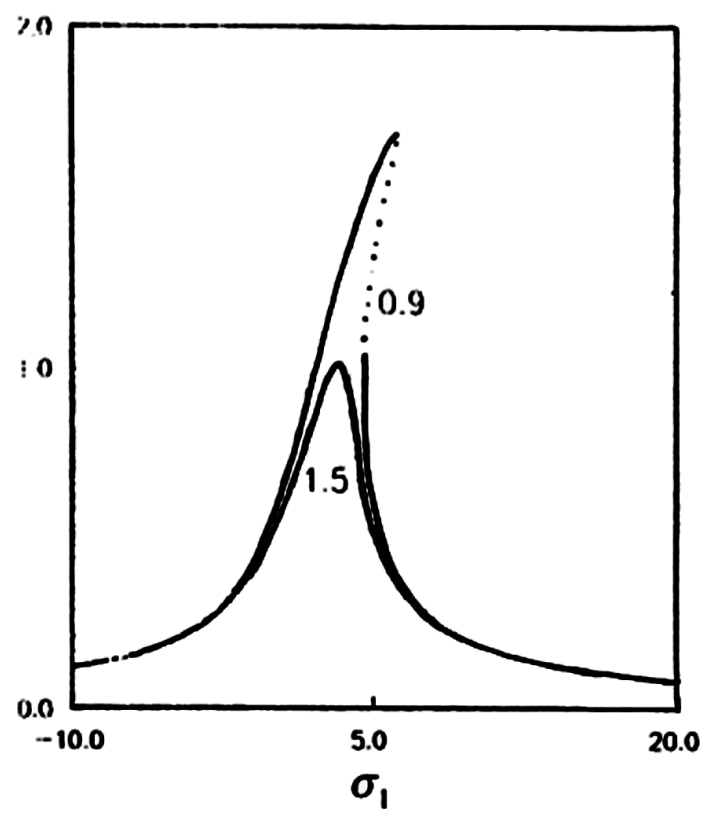

Fig. 3. Frequency-response curve for superharmonic resonance for increasing the damping factor $\mu$ on the response when $\mu$ takes the values 0.9 and 1.5. (-) Stable and $(\cdots)$ unstable.

such that the upper branch has a stable solution and the lower one has stable and unstable solutions. As $\sigma_{1}$ decreases 4-20, the response amplitude $r$ loses stability via a saddle node bifurcation. The bending of 


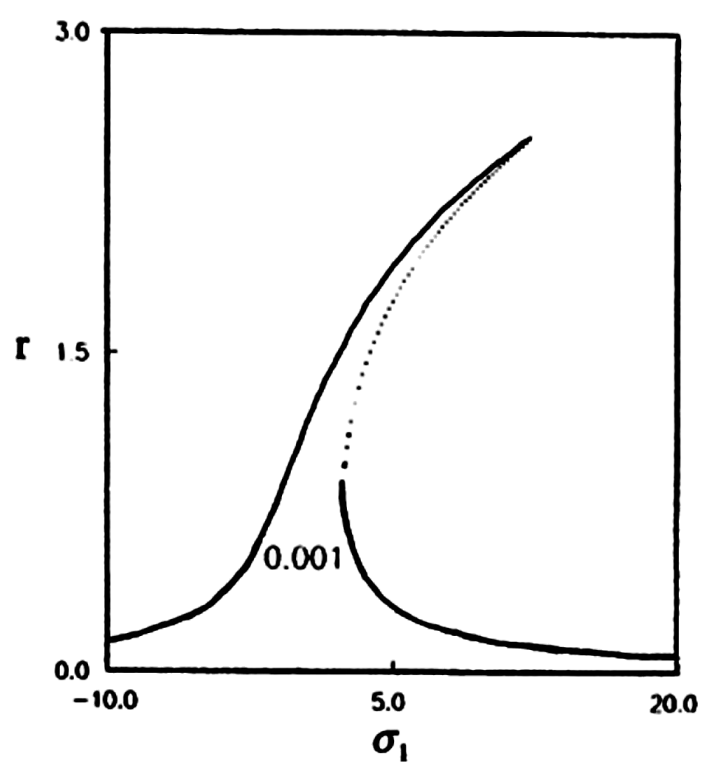

Fig. 4. Frequency-response curve for superharmonic resonance for decreasing the coefficient of cubic nonlinearity $\alpha$ on the response when $\alpha=0.001$. (-) Stable and ( . . ) unstable.

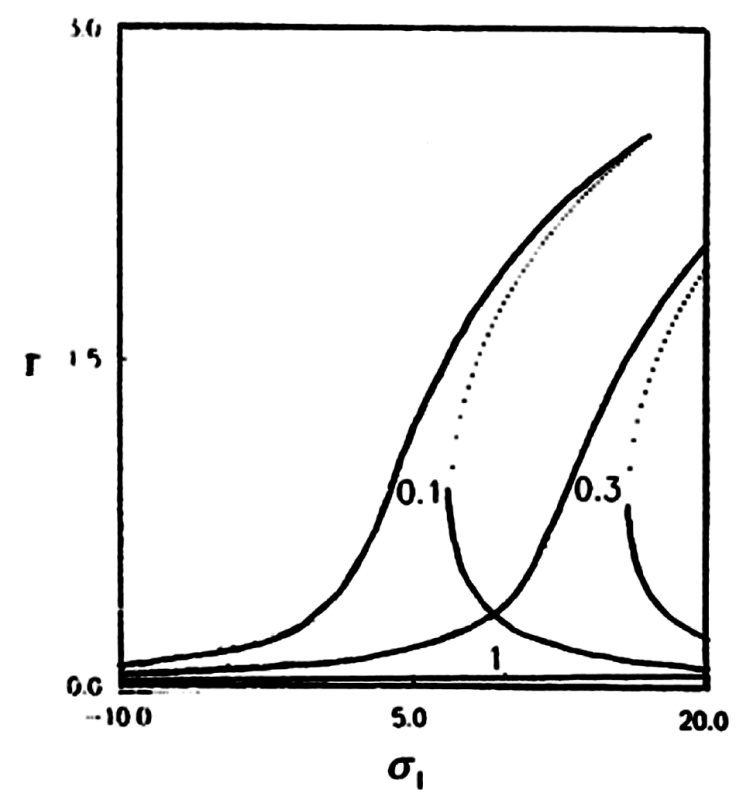

Fig. 5. Frequency-response curve for superharmonic resonance for increasing the coefficient of cubic nonlinearity $\alpha$ on the response when $\alpha$ takes the values 0.1 and 0.3. (-) Stable and $(\cdots)$ unstable.

the curves leads to multi-valued solutions and hence to the jump phenomenon. As the coefficient of the damping factor $\mu$ decreases down to 0.01 , the two branches separate from each other and have larger magnitudes.

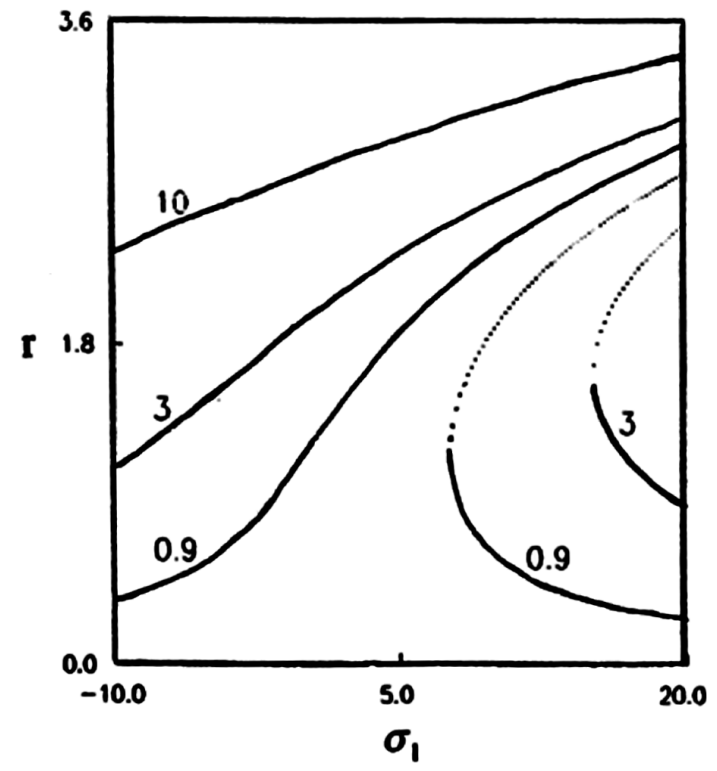

Fig. 6. Frequency-response curve for superharmonic resonance for increasing the coefficient of quartic nonlinearity $\beta$ on the response when $\beta$ takes the values $0.9,3$ and 10. (-) Stable and $(\ldots)$ unstable.

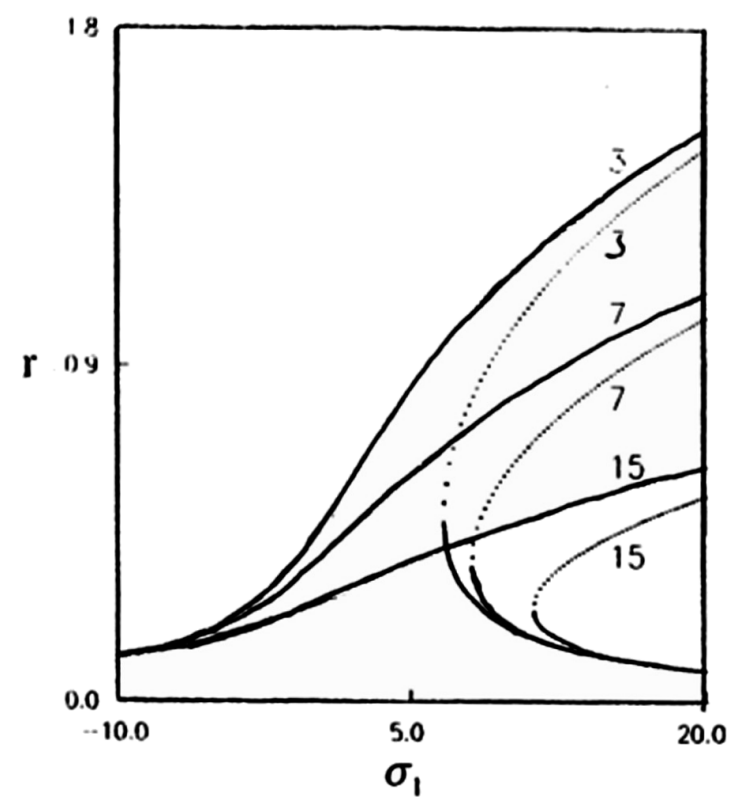

Fig. 7. Frequency-response curve for superharmonic resonance for increasing the coefficient of quaitic nonlinearity $\gamma$ on the response when $\gamma$ takes the values 3,7 and 15 . (-) Stable and $(\cdots)$ unstable.

The multi-valued zone grows. As $\sigma_{1}$ decreases through the interval 5-20, the response amplitude loses stability via a saddle node bifurcation and the bending 


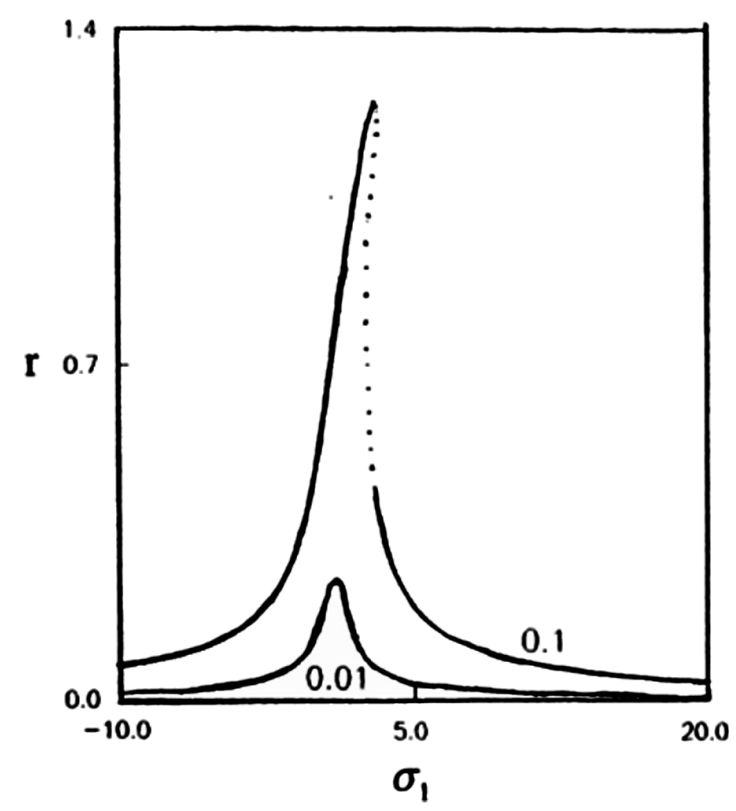

Fig. 8. Frequency-response curve for superharmonic resonance for decreasing the coefficient of external excitation $f$ on the response when $f$ takes the values 0.1 and 0.01 . (-) Stable and $(\cdots)$ unstable.

leads to the jump phenomenon (Fig. 2). For a damping factor $\mu$, which is larger than in Fig. 1, we note that the bending is contracted, which leads to a smaller range of multi-valuedness. The response amplitude has a lower magnitude. There exists a saddle node bifurcation and again the jump phenomenon. As $\mu$ increases up to 1.5 , the multi-valuedness disappears and the response amplitude is stable and single-valued (Fig. 3). When the coefficient of the cubic nonlinearity $\alpha$ decreased down to 0.001 , we observe that the bending of the response amplitude shifts towards the left and there exists a saddle node bifurcation and also the jump phenomenon (Fig. 4). When $\alpha$ is chosen as 0.01 , the bending of the response amplitude shifts to the right. As $\sigma_{1}$ decreases through the interval 4-20, the response amplitude loses stability via a saddle node bifurcation. The bending of the curves leads to the jump phenomenon. As $\alpha=0.3$, the two branches separate from each other and have smaller magnitudes. The bending decreases which leads to decreasing $\gamma$ in the range of multi-valuedness and there exists a saddle node bifurcation and the jump phenomenon (Fig. 5). For a smaller value of the coefficient of the quartic nonlinearity $\beta$, we note that the two branches separate from each other and the two branches shift upwards to larger magnitudes. There exists a saddle node bifur-

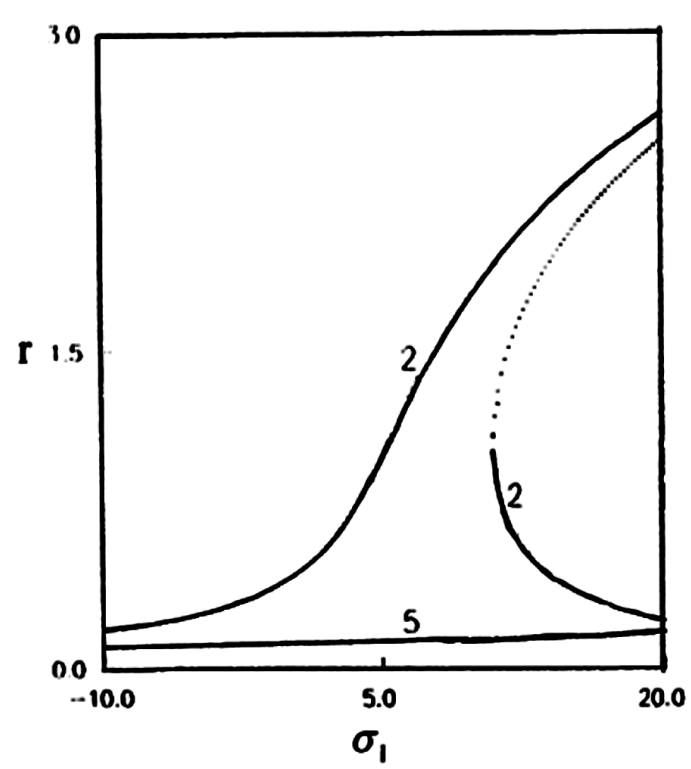

Fig. 9. Frequency-response curve for superharmonic resonance for increasing the coefficient of external excitation $f$ on the response when $f$ takes the values 2 and 5. (-) Stable and $(\cdots)$ unstable.

cation and the jump phenomenon. As $\beta=3$, we note that the upper branch has an increased stable magnitude and the lower branch is contracted, implying a smaller $r$ in the range of multi-valuedness. When $\beta$ increases further up to 10 , the lower branch disappears and the response amplitude is a single-valued curve with smaller stable magnitude (Fig. 6). As the coefficient of the quintic nonlinearity $\gamma$ decreases, we observe that the two branches separate from each other and the two branches move to the right with smaller magnitudes. When $\sigma_{1}$ runs through the interval 6-20, the response amplitude $r$ loses its stability via a saddle node bifurcation and the bending again leads to the jump phenomenon. As $\gamma$ takes the values 7 and 15 the response amplitude has the same magnitudes for a small interval of $\sigma_{1}$ and after this interval it is shifted downwards with smaller magnitudes, respectively. The multi-valued curve is contracted and has smaller $r$ values in the region of multi-valuedness, respectively (Fig. 7). When the coefficient of quintic nonlinearity $\gamma$ becomes smaller, we observe that the bending contracts and the region of multi-valuedness is smaller. When $\sigma_{1}$ runs through the interval 3-20, the response amplitude loses its stability via a saddle node bifurcation and there exists the jump phenomenon. For $\gamma$ as small as 0.01 , the multi-valuedness disappears and the response amplitude is a stable single-valued curve 


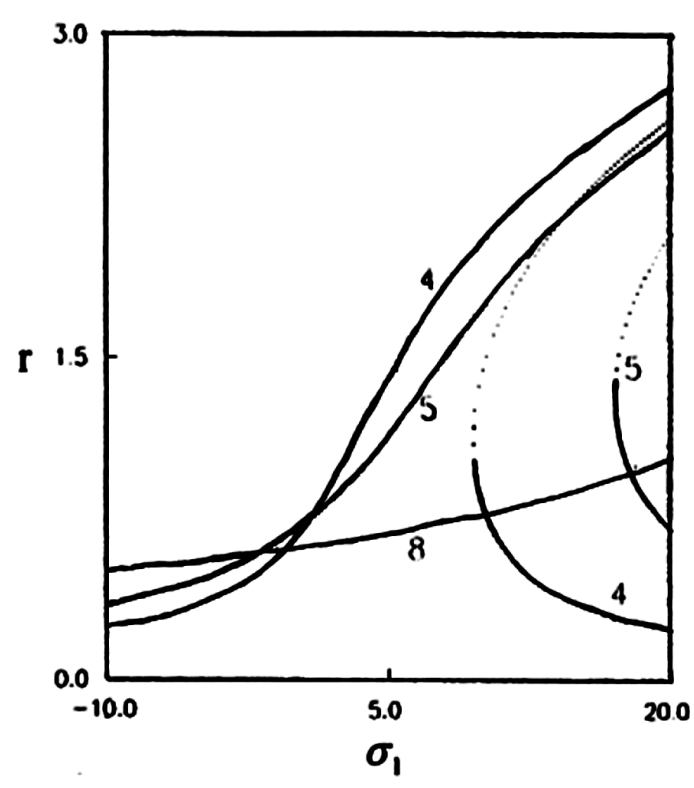

Fig. 10. Frequency-response curve for sub-superharmonic resonance for increasing the coefficient of external excitation $g$ on the response when $g$ takes the values 4,5 and 8 . (一) Stable and $(\cdots)$ unstable.

(Fig. 8). For increasing the coefficient of the external excitation $f$, we note that the two branches separate from each other and shift to the right. There exists a saddle node bifurcation and the jump phenomenon. When $f=3$, we note that the lower branch disappears and the upper branch moves downwards, becomes smaller and stable magnitudes (Fig. 9). When the coefficient of the external excitation $g$ is increased, we observe that the two branches separate from each other and the zone of multi-valuedness develops for larger values of $\sigma_{1}$. The response amplitude has an increased magnitude and as $\sigma_{1}$ decreases, the response amplitude loses stability via a saddle node bifurcation. The bending leads to the jump phenomenon. As $g=5$, the lower branch is contracted and leads to decreasing $r$ in the range of multi-valuedness. There exists a saddle node bifurcation as well as the jump phenomenon. For further increasing of $g$ up to 8, the lower branch disappears and the response amplitude is a stable, singlevalued curve (Fig. 10).

Figures 11-18 represent the frequency-response curves of the second case (sub-superharmonic resonance). The effect of the different parameters on the system are investigated. In Table 2 the investigated ranges of the considered parameters are summarized.

In Fig. 11, we observe that the response amplitude starts with a minimum value and is a multi-valued
Table 2. Ranges of the different parameters investigated.

\begin{tabular}{lll}
\hline Figure number & Investigated parameter & Range \\
\hline 11,12 & Damping factor $\mu$ & 1.2 to 0.1 \\
13,14 & Coefficient of cubic nonlinearity $\alpha$ & 0.28 to 0.05 \\
15 & Coefficient of quintic nonlinearity $\gamma$ & 0.5 to 7 \\
16,17 & Coefficient of external excitation $f$ & 1.8 to 0.5 \\
18 & Coefficient of external excitation $g$ & 3 to 4.3 \\
\hline
\end{tabular}

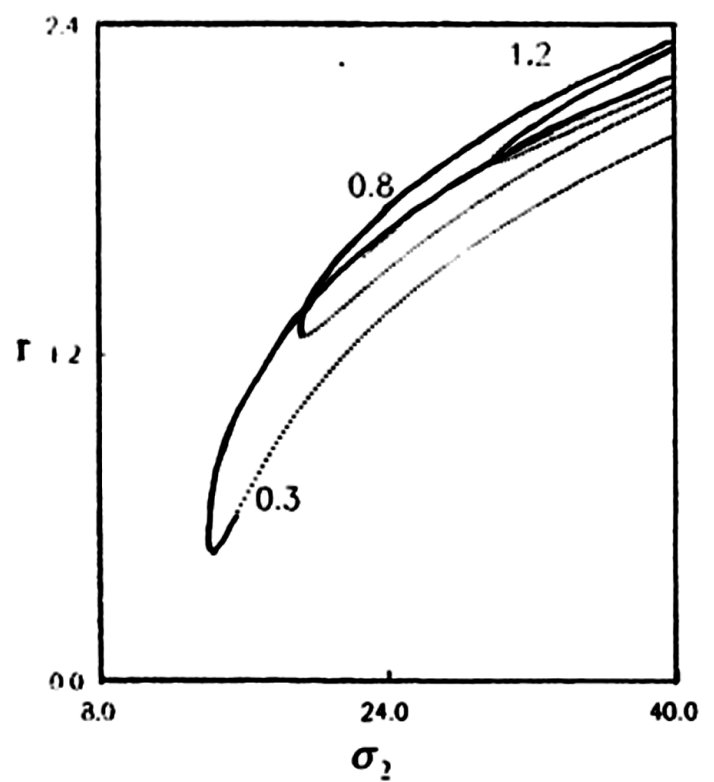

Fig. 11. Frequency-response curve for sub-superharmonic resonance for the parameters $\mu=0.3, \alpha=0.1, \gamma=0.5$, $f=1, g=3$ and the effect of increasing the damping factor $\mu$ on the response when $\mu$ takes the values 0.8 and 1.2. (-) Stable and (...) unstable.

curve, which is closed from below and open from above. The upper branch corresponds to a stable solution and the lower branch has stable and unstable solutions. As $\sigma_{2}$ scans the interval 14-20, the lower branch loses its stability via a saddle node bifurcation. When the damping factor $\mu$ is chosen as 0.8 or 1.2 the response amplitudes are contracted and have increased minimum values, respectively, which imply smaller values in the regions of definition, multi-valuedness and stability. There exist saddle node bifurcations. For choosing the damping factor $\mu$ as 0.1 , the response amplitude has a smaller minimum value, and the range of definition of multi-valuedness and stability are larger. The lower branch has unstable solutions and there exists a saddle node bifurcation (Fig. 12). As the coefficient of the cubic nonlinearity $\alpha$ is increased, i. e $\alpha$ takes the values 0.2 and 0.28 , the minimum values of the response amplitude shift to the right with decreased magnitudes and give smaller values in the ranges of 


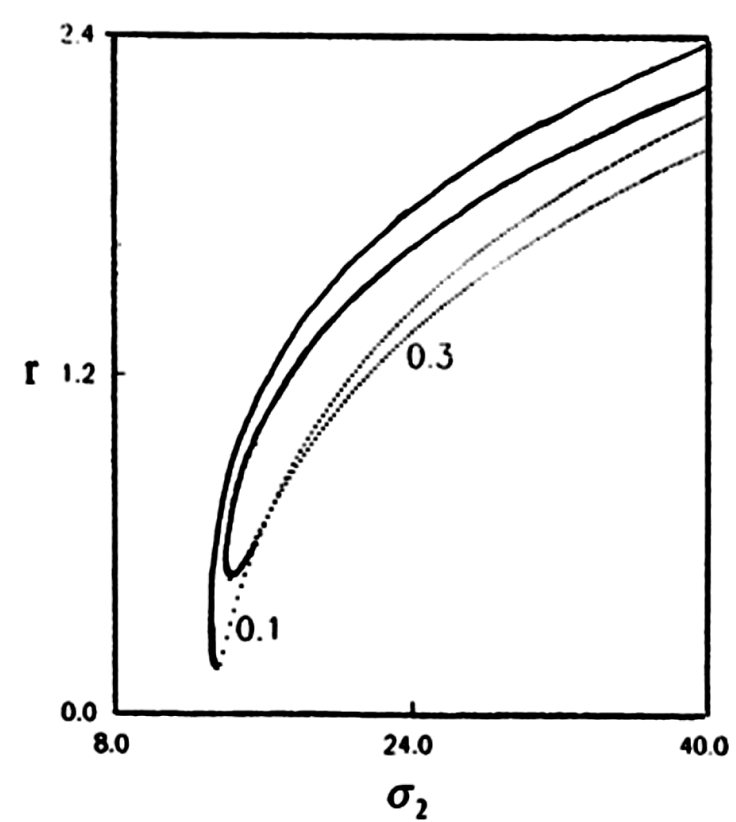

Fig. 12. Frequency-response curve for sub-superharmonic resonance for decreasing the damping factor $\mu$ on the response when $\mu$ takes the values 0.3 and 0.1. (-) Stable and $(\cdots)$ unstable.

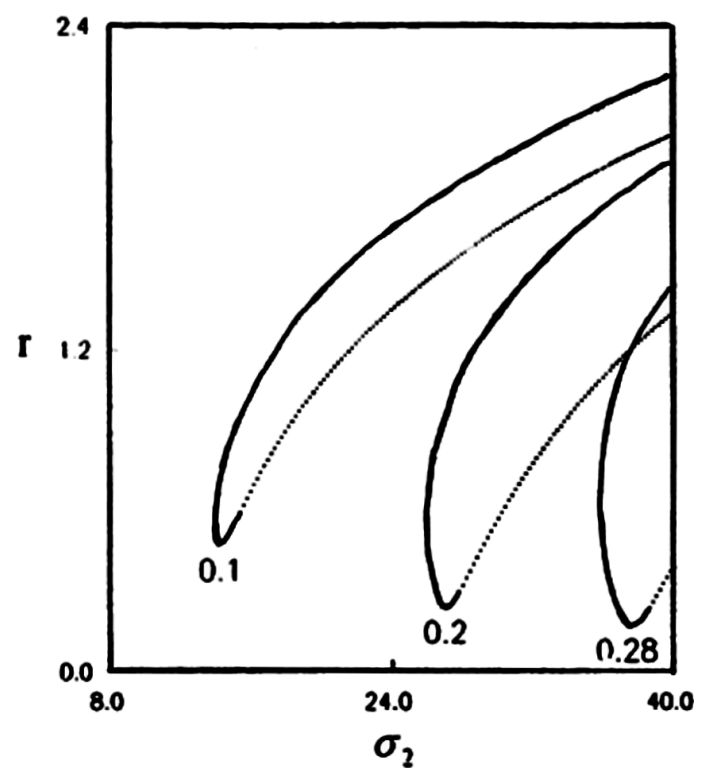

Fig. 13. Frequency-response curve for sub-superharmonic resonance for increasing the coefficient of cubic nonlinearity $\alpha$ on the response when $\alpha$ takes the values $0.1,0.2$ and 0.28 $($ - Stable and $(\cdots)$ unstable.

definition, multi-valuedness and stability. There exists a saddle node bifurcation in the lower branch (Fig. 13).

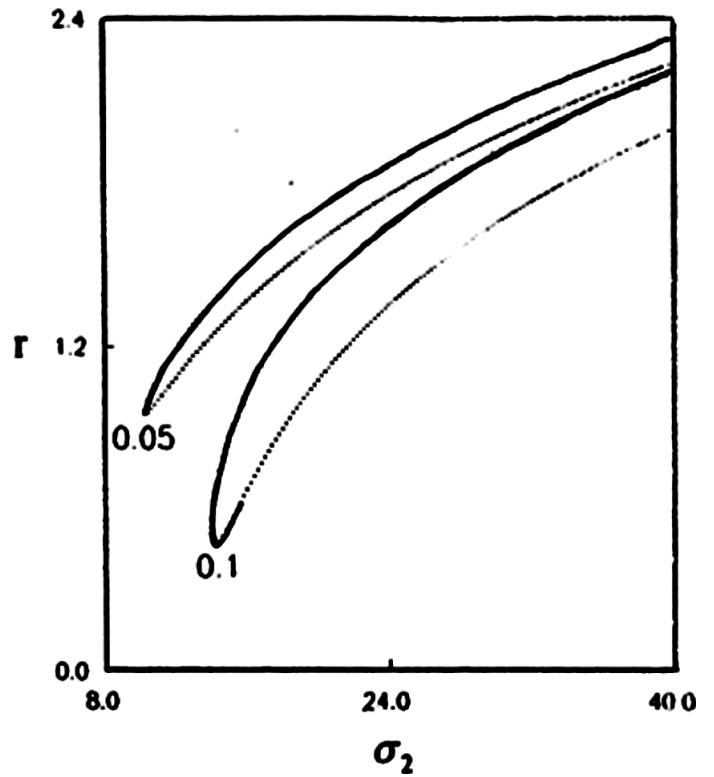

Fig. 14. Frequency-response curve for sub-superharmonic resonance for decreasing the coefficient of cubic nonlinearity $\alpha$ on the response when $\alpha$ takes the values 0.1 and 0.05 . (-) Stable and $(\cdots)$ unstable.

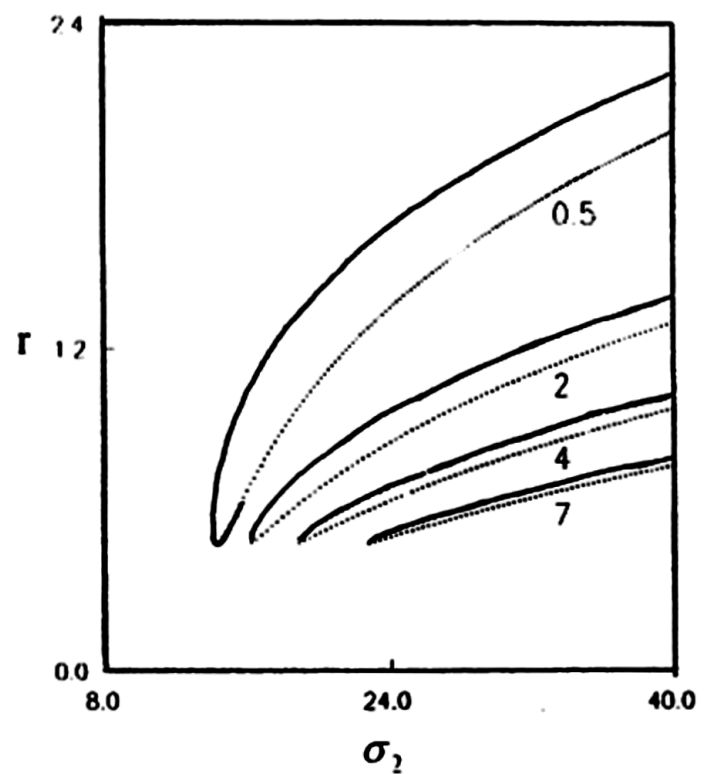

Fig. 15. Frequency-response curve for sub-superharmonic resonance for increasing the coefficient of quintic nonlinearity $\gamma$ on the response when $\gamma$ takes the values $0.5,2,4$ and 7 . (-) Stable and (..) unstable.

When $\alpha$ is decreased down to 0.05 , the response amplitude becomes larger and has a larger minimum value. The regions of definition, multi-valuedness and 


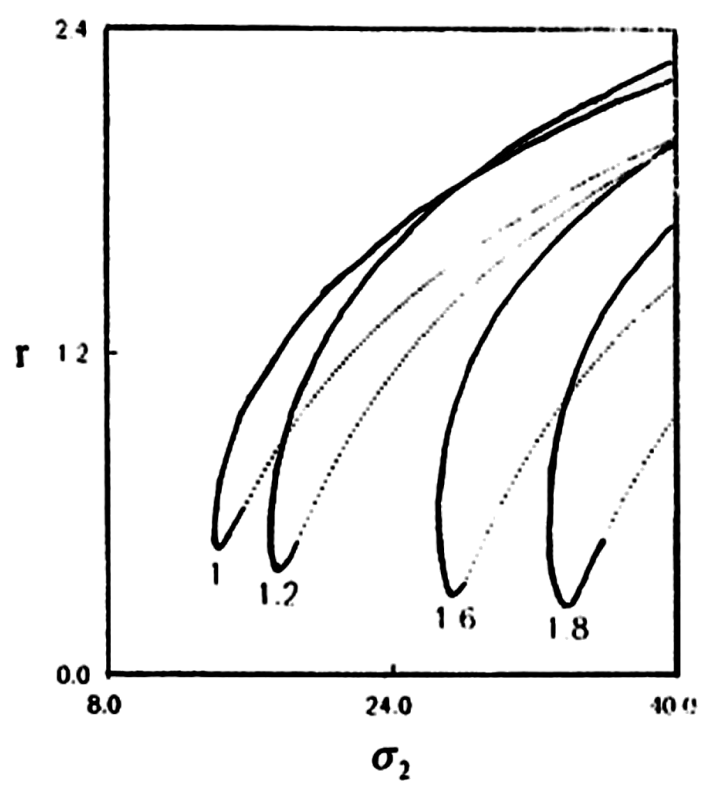

Fig. 16. Frequency-response curve for sub-superharmonic resonance for increasing the coefficient of external excitation $f$ on the response when $f$ takes the values $1,1.2,1.6$ and 1.8. (-) Stable and (..) unstable.

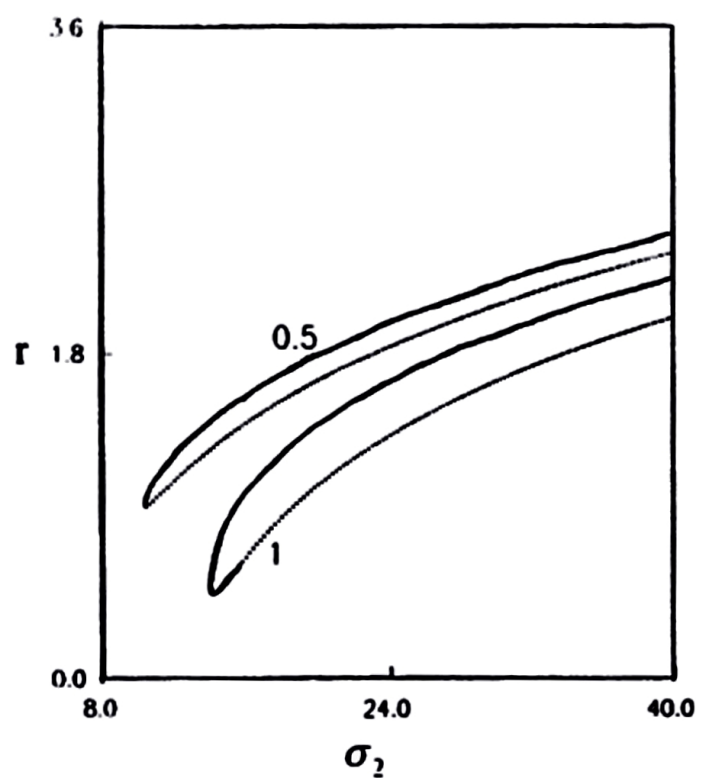

Fig. 17. Frequency-response curve for sub-superharmonic resonance for decreasing the coefficient of external excitation $f$ on the response when $f$ takes the values 1 and 0.5 . $(-)$ Stable and $(\cdots)$ unstable.

stability become larger. The lower branch has an unstable solution and there exists a saddle node bifurcation (Fig. 14). As the coefficient of quintic nonlin-

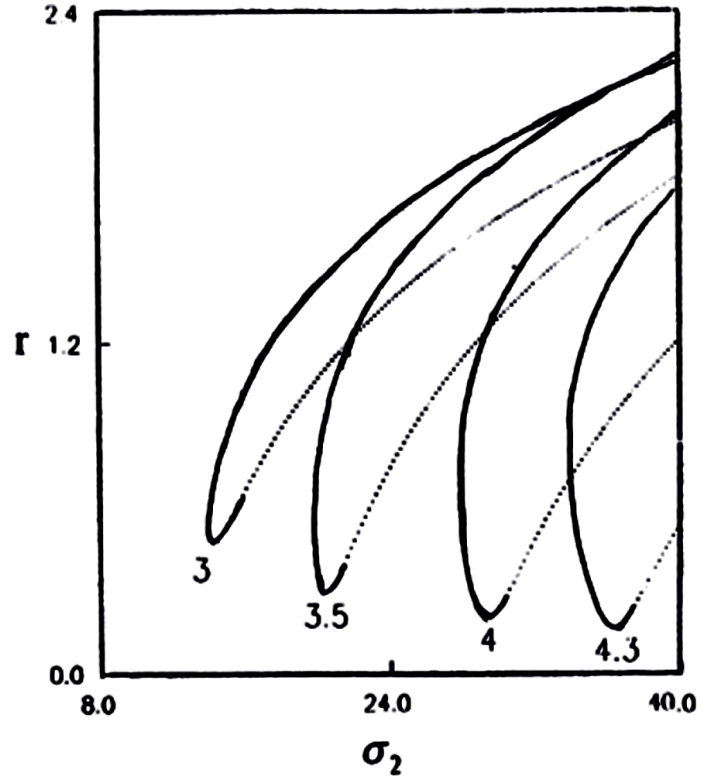

Fig. 18. Frequency-response curve for sub-superharmonic resonance for increasing the coefficient of external excitation $g$ on the response when $g$ takes the values 3, 3.5, 4 and 4.3. $($ - Stable and $(\cdots)$ unstable.

earity is increased, we observe that the response amplitude is contracted and shifts downwards to smaller magnitudes and minimum values for large values of $\sigma_{2}$. The ranges of definition, multi-valuedness and stability are increased. The lower branch has unstable solutions, there exists a saddle node bifurcation (Fig. 15). When the coefficient of the external excitation $f$ increases, we observe that the response amplitude shifts towards the right and the minimum value goes downwards. The ranges of definition, multi-valuedness and stability become smaller. The lower branch has stable and unstable solutions, and there exists a saddle node bifurcation in the lower branch (Fig. 16). When the coefficient of the external excitation $f$ is increased, we get the same variation as in Fig. 4 such that the lower branch has unstable solutions and there exists a saddle node bifurcation (Fig. 17). Similar as in Fig. 9 we have shown the variations if the coefficient of the external excitation $g$ grows, we get similar variations if $g$ takes the values 3.5, 4, and 4.3, as shown in Figure 18.

\section{Concluding Remarks}

The response of single-degree-of-freedom with cubic, quartic and quintic nonlinearities to a modulated high-frequency input was studied. The method of mul- 
tiple scales is used to derive a second-order equation governing the components of the response of the system near its natural frequency. In this equation, the amplitude of the excitation, which is a function of time, appears as parametric excitation. The result of the perturbation analysis was used to study the response of the system to a constant amplitude and harmonically modulated excitations. For harmonically modulated excitations, various resonances occur, and these were shown to correspond to external combination resonances. These examples are fairly straightforward, but more interesting cases, involving a periodic modulation, could be studied using the analysis in this paper. Two coupled first-order ordinary differential equations describe the evolution of the amplitude and phase with damping, nonlinearities and resonances. The evolution equations are used to determine the steady-state motions and representative frequency-response curves are presented for each resonance. The stability of the steady-state solution is investigated.

The present results reveal the following features of the steady-state response:

[1] A. H. Nayfeh, J.F. Nayfeh, and D. T. Mook, Nonlinear Dynamics 3, 145 (1992).

[2] Q. S. Lu, C. W. S. To, and K. L. Huang, Dyn. Stability Syst. 8, 31 (1993).

[3] A. H. Nayfeh, S. A. Nayfeh, and M. Pakemirli, Nonlinear Dynamics and Stochastic Mechanics (Eds. W. Kliemann and N. Sri Namachchivaya), CRC Press, Boca Raton, FL 1995, pp. 175.

[4] S. A. Nayfeh and A. H. Nayfeh, Nonlinear Dynamics 7, 301 (1995).

[5] J. J. Thomsen, J. Sound Vibr. 197, 403 (1996).

[6] T. J. Anderson, A.H. Nayfeh, and B. Balachandran, J. Sound Vib. 118, 21 (1996).

[7] A. N. Deryugin, A. Y. Loskutov, and V. M. Tereshko, Chaos, Solitons and Fractals 7, 1555 (1996).

[8] H. Yabuno, Nonlinear Dynamics 12, 236 (1997).

[9] J. F. Wilson, Int. J. Non-Linear Mech. 33, 189 (1998).

[10] E. E. Zadeh and N. Galili, Int. J. Non-Linear Mech. 33, 765 (1998).

[11] A. H. Nayfeh, Nonlinear Dynamics 16, 105 (1998).

[12] G.-M. Ge, C.-I. Lee, H.-H. Chen, and S.-C. Lee, J. Sound Vibr. 217, 807 (1998).

[13] A. Raghothama and S. Narayanan, J. Sound Vibr. 22, 469 (1999).

[14] W. Kreider and A. H. Nayfeh, Nonlinear Dynamics 15, 155 (1998).

[15] W. Lacarbonara and A. H. Nayfeh, Nonlinear Dynamics 17, 95 (1998).
In the case of superharmonic resonance we observe that the bending of the response curves leads to multivalued solutions and hence to the jump phenomenon. The two branches of the response amplitude diverge from each other on decreasing the parameter $\mu$ and increasing the parameters $\alpha, \beta, \gamma, f$ and $g$, respectively. The region of multi-valued solutions is increased on increasing the damping factor $\mu$. The response amplitude is stable and single-valued, when the parameters $\mu, \alpha$, $\beta, f$, and $g$ take the values $1.5,10,1,5$ and 8 , respectively.

In the case of a sub-superharmonic resonance we note that the bending of the response amplitude begins with a minimum value and is a multi-valued curve. The minimum value of the response amplitude shifts to the right with decreased magnitude for increasing the parameters $\alpha, f$ and $g$, respectively. The regions of definition, multi-valuedness and stability are decreased for increasing the parameters $\alpha, \gamma, f$, and $g$. The response amplitude is contracted and shifts downwards with decreased magnitudes for increasing the coefficient of quintic nonlinearity $\gamma$.

[16] S. S. Oueini and A.H. Nayfeh, Nonlinear Dynamics 224, 33 (1999).

[17] A. F. El-Bassiouny and H. M. Abdelhafez, Math. Comput. Simulation 57, 61 (2001).

[18] A. Maccari, Nonlinear Dynamics 26, 105 (2001).

[19] Y. O. El-Dib, Chaos, Solitons and Fractals 12, 705 (2001).

[20] M. Eissa and A. F. El-Bassiouny, Appl. Math. Comput. 134, 243 (2003).

[21] K. Alsaif, Solitons and Fractals 15, 619 (2003).

[22] A. F. El-Bassiouny and M. Eissa, Appl. Math. Comput. 139, 1 (2003).

[23] X. Wu and Y.P. Li, Mech. Res. Commun. 31, 519 (2004).

[24] A. R. F. Elhefnawy and A. F. El-Bassiouny, Chaos, Solitons and Fractals 23, 289 (2004).

[25] A. Maccari, Chaos, Solitons and Fractals 22, 25 (2004).

[26] A.F. El-Bassiouny, Mech. Res. Commun. 32, 337 (2005).

[27] H. Cao, Chaos, Solitons and Fractals 24, 1387 (2005).

[28] Z. Jing and R. Wang, Chaos, Solitons and Fractals 23, 399 (2005).

[29] M. Pakemirli, S. A. Nayfeh, and A. H. Nayfeh, Nonlinear Dynamics 8, 65 (1995).

[30] A. Maccari, Int. J. Non-Linear Mech. 36, 335 (2001).

[31] A.F. El-Bassiouny, Appl. Math. Comput. 134, 217 (2003). 
[32] A.M. Elnaggar and A.F. El-Bassiouny, Appl. Math. Comput. 139, 179 (2003).

[33] Q. Bi, Int. J. Non-Linear Mech. 39, 33 (2004).

[34] A.F. El-Bassiouny and M. Eissa, Phys. Scr. 70, 101 (2004).

[35] A. F. El-Bassiouny, Phys. Scr. 71, 561 (2005).
[36] A. F. El-Bassiouny, Phys. Scr. 72, 203 (2005).

[37] A. F. El-Bassiouny, Phys. Scr. 72, 132 (2005).

[38] A. H. Nayfeh, Introduction to Perturbation Techniques, Wiley-Interscience, New York 1981.

[39] A.F. El-Bassiouny, Solitons and Fractals 30, 1098 (2006). 